\title{
Techniques for combinatorial molecular beam epitaxy
}

\author{
Frank Tsuia) and Liang He \\ Department of Physics and Astronomy, University of North Carolina at Chapel Hill, North Carolina 27599
}

(Received 19 November 2004; accepted 30 January 2005; published online 18 May 2005)

\begin{abstract}
Basic considerations for implementing combinatorial approach to molecular beam epitaxy (MBE) are discussed, focusing on the key issues relevant to conventional MBE synthesis using solid sources and characterization. The primary objective for implementing combinatorial approach is to make MBE do more, more able to carry out controlled and systematic work in large parameter space, without sacrificing any existing capabilities of conventional MBE. Methods for accomplishing this by integrating current instrumentation technology are described.

(C) 2005 American Institute of Physics. [DOI: 10.1063/1.1905967]
\end{abstract}

\section{INTRODUCTION}

Since its early development during the 1970s, molecular beam epitaxy (MBE) has been the standard for synthesis of highest quality coherent epitaxial films and heterostructures, ${ }^{1}$ from quantum wells to two-dimensional (2D) electron systems, and from magnetic superlattices to diluted magnetic semiconductors. It is a collection of techniques that can be employed to control and characterize the epitaxial growth processes under UHV conditions. Among these are a set of tightly controlled "knobs" called growth parameters, such as what kind of substrate to use and how to prepare it, and growth temperatures and rates, and an array of in-situ characterization techniques, including real-time reflection highenergy electron diffraction (RHEED) for examining surface structures during growth. The large number of controlled parameters makes it possible to tailor materials and properties on atomic scale, and to examine these by systematically varying one or several of the parameters, while keeping the rest of them fixed.

Conventional MBE produces one sample on one substrate. This process involves a sequence of meticulously executed steps, typically starting from substrate preparation, and stabilizing and calibrating evaporation sources, to preparation of a buffer layer, and then the growth of the film or heterosturcture, and finally finishing-up with a passivation/ cap layer. The entire process usually takes many hours to complete, so to produce one sample in a day is what one would normally expect. Suffice to say, it is a slow and tedious process, such that using it to explore complex materials systems, such as ternary and quaternary alloys, is extremely difficult and requires significant resources.

In this paper we describe the basic instrumentation, key technical considerations and approaches for adapting combinatorial approach to conventional MBE for exploring complex epitaxial systems. We show that it is possible to explore large parameter space systematically using only a few actual samples, without sacrificing the control and other key char-

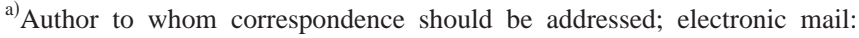
ftsui@physics.unc.edu
}

acteristics of MBE. The development and integration of the techniques have been motivated by the success of combinatorial approach in other physical vapor deposition processes. ${ }^{2-6}$ Combinatorial MBE has been used to study synthesis and characterization of ternary systems containing group IV and transition metal elements, ${ }^{7}$ which led to the discovery of Ge-based magnetic semiconductor epitaxial films and heterostructures. ${ }^{8-10}$

\section{BASIC TECHNIQUES FOR IMPLEMENTING COMBINATORIAL MOLECULAR BEAM EPITAXY}

The essence of a controlled combinatorial vapor deposition process is the ability to place a tailored set of materials parameters, the "variables," such as composition, film thickness, and epitaxial strain, onto a substrate and to examine the corresponding properties systematically in real time. Each combinatorial sample can have one, or two, or even multiple variables "programmed" into it, effectively slicing through the parameter space and thus enabling investigation of the corresponding behaviors systematically. In order to do this using MBE, the basic requirements are a sample stage that can be manipulated with precision, a system of masks that can place the flux at chosen locations on a substrate precisely, instruments that can monitor and control deposition rates of a number of sources, and techniques to characterize properties in real time.

The integration and operation of these components are illustrated schematically in Fig. 1. The sample manipulator can move the substrate into proper position and proper inplane orientation with respect to the deposition masks. The azimuthal crystallographic axis of the substrate can be determined and aligned precisely by the corresponding RHEED pattern, which can be recorded in real time using a chargecoupled device $(\mathrm{CCD})$ camera and analyzed. A profile of materials parameters, the variables, such as a submonolayer "wedge" or a stack of different wedges, can be "programmed" onto the substrate by sequentially moving the masks across the substrate during a deposition sequence involving several evaporation sources. The movement of the masks and the associated "exposure" time can be controlled by the use of real-time flux monitors, e.g., atomic absorption 


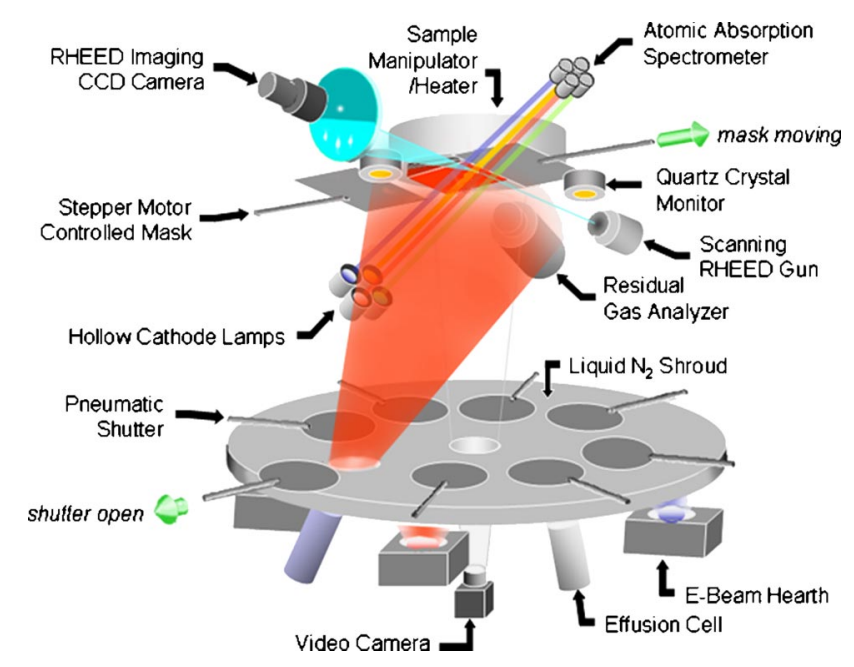

FIG. 1. (Color online) Schematic diagram of a combinatorial MBE system. The diagram depicts the process of depositing a linear composition profile (in red) using a combination of mask and pneumatic shutter (the green arrows). The speed of the mask and the associated shutter exposure time are determined by the signal from atomic absorption (AA) flux monitors. The RHEED beam can be scanned across the sample during growth and annealing with the diffraction patterns on the phosphors screen captured by a CCD camera.

(AA) spectroscopy monitors. It is critical to examine surface properties during growth using scanning and imaging techniques, including scanning RHEED, in order to understand and optimize the growth processes as a function of the programmed variable(s).

In the following sections, we first describe the basic materials parameters that can be programmed into a combinatorial MBE sample and the prospects for extending into additional ones. We then detail various schemes for masks and mask alignment, and followed by options for real-time flux monitoring. Finally, techniques for real-time characterization of properties are discussed.

\section{A. Considerations for combinatorial MBE samples}

The variables, that are important to $\mathrm{MBE}$ growth and are also readily programmable into a combinatorial $\mathrm{MBE}$ sample, include film thickness, composition, lattice strain, and substrate miscut. Other important variables that are currently not fully implemented are growth temperature and flux. Details about these variables are discussed as follows.

A composition/thickness profile can be produced by depositing multilayers of wedges of atomic layers. When the thicknesses of the individual layers are more than several atomic layers, multilayers with different components and different layer thicknesses can be studied systematically. In contrast when they are reduced to atomic scale, alloys with varying concentrations can be made and studied. The multilayer method is not only easy to implement in $\mathrm{MBE}$ systems, but also offers unique opportunity to tailor/sequence the nonequilibrium synthesis of alloys. For example, it can be used to promote a particular stacking along the growth direction, or to tailor surfaces and interfaces to obtain desired effects, such as an ohmic interface or a passivated surface.

Strain effects can be studied by using a combinatorial buffer layer with varying lattice constants as the growth tem-

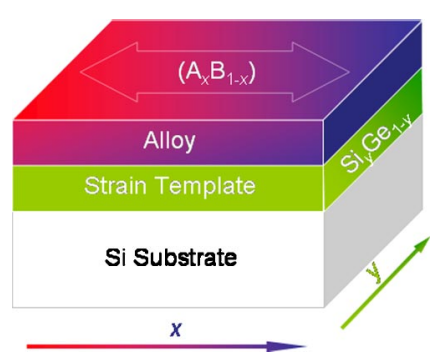

FIG. 2. (Color online) Schematic diagram of a combinatorial sample, $A_{x} B_{1-x}$, tailored for studying dependence of properties on the composition$x$ axis, and on epitaxial strain provided by the $\mathrm{Si}_{y} \mathrm{Ge}_{1-y}$ template- $y$ axis. Thus the lattice constant can be varied continuously from that of Si to Ge. Growth and properties of the alloy film can be examined systematically.

plate. As illustrated in Fig. 2, alloys can be used to fine-tune the lattice constants, for example, $\mathrm{Si}_{x} \mathrm{Ge}_{1-x}$ alloys for group IV systems, ${ }^{11}$ and $\mathrm{Y}_{x} \mathrm{Lu}_{1-x}$ alloys for the rare earths. ${ }^{12}$ Effects of substrate miscut can be examined by using special substrates that are polished with continuously changing miscut, the "curved" substrates.

Variation of growth temperature and flux would require controlled temperature and flux gradients that are very difficult to implement in standard MBE systems, since uniformity and stability of both of these are the built-in premium for the controlled operation of MBE. Possible solutions include to implement a multizone radiation heater or laser heating similar to that in laser $\mathrm{MBE}^{6}{ }^{6}$ and to augment the relative alignment between the substrate and the atomic beam, ${ }^{13}$ such that the substrate is placed at the sloping/fringe part of the flux, instead of the peak/uniform part. While these and other options are being actively pursued, more convenient approaches have been used to explore these dependences. One of these is to sequentially grow and study many small patches of films on a single substrate using precision masks at different growth temperatures and growth rates. Since substrate and buffer preparations, sample introduction and transfer, source calibration, etc., are rather time consuming, this sequential method can still substantially enhance the rate at which the number of combinations are produced and studied. The size of the patches, and therefore the quantity that can be put on each substrate, are determined by the spatial spread of the patches and the size and alignment of the measurement probes, e.g., RHEED. These effects are discussed in the next section, and currently we are able to grow and characterize 10 patches per $\mathrm{cm}$ routinely.

\section{B. Deposition mask and mask alignment}

As described above, a multilayer of wedge shaped layers can be employed to deposit the variables onto a substrate. The masks, mounted on stepper motor controlled precision feedthroughs, can be designed to form different shapes and sizes, such as an edge or a slit, so by moving them across a substrate during evaporation, either discrete or continuously varying compositional profiles can be produced on the substrate. A combinatorial sample with one variable programmed along one in-plane axis of the substrate is called a binary sample, and a sample with two variables on the plane 


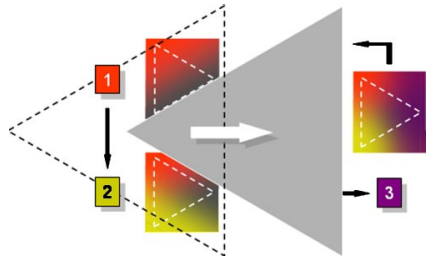

FIG. 3. (Color online) A scheme for depositing a ternary sample using a single mask on a linear motion feedthrough and translational motion of the substrate, sequentially from position 1 to position 3 . The materials to be deposited are correspondingly labeled as material 1 to 3 . The intended ternary region is outlined by a triangle of white dashed lines on the substrate. The film thicknesses, i.e., wedges, are depicted by the color gradient with the bright colors corresponding to thicker parts of the films.

of the substrate is a ternary one. For example, a ternary sample can contain the complete compositional combinations of a ternary alloy. ${ }^{7}$

In combinatorial MBE it is important to have economical/efficient mask designs and masking schemes, so that minimum number of masks and least amount of movement are required for the growth process. There are several reasons for this. First, UHV feedthroughs with stepper motors are typically not designed for fast motion and large number of repetitions, particularly for some of the precision rotational feedthroughs. Second, a lot of movements with many motors are prone to cause slippage in positioning and even failure in mechanical and vacuum components. Overall a lot of slow movements not only prolong the growth process unnecessarily, but also can make it imprecise.

In Fig. 3 we illustrate one efficient scheme to make a ternary sample with a continuous composition profile, using only one mask mounted on a linear motion feedthrough and one translational motion of the sample stage. The intended ternary phase diagram on the substrate is indicated by a triangle, the "ternary triangle," outlined by the white dashed line in Fig. 3. This has been used to produce ternary phase diagrams of $\mathrm{CoMnGe},{ }^{14}$ and is a variation of another proposed scheme for laser $\mathrm{MBE}^{15}$ and an improvement from one that requires sample rotation. ${ }^{4,6,7}$ Procedure for depositing a trilayer of submonolayer wedges is described as follows. At position 1, by moving the mask from one of apexes of the ternary triangle to its respective baseline during deposition, a wedge layer of material 1 can be produced across the ternary triangle accordingly. By moving the substrate to position 2, a wedge of material 2 from a different apex of the ternary triangle to its baseline can be produced using a different edge of the mask. Subsequently, by moving the substrate to position 3 and using yet another different edge of the mask, a wedge of material 3 can be deposited, thus completing the ternary trilayer. A thick combinatorial film can therefore be built-up by repeating the trilayers.

In order to execute a masking scheme, the relative positions between the substrate and the mask need to be determined. Since the sources are necessarily located at different positions in the growth system (see Fig. 1) and the masks are not making contact with the substrate, the shadows cast by the masks are source dependent, so the substrate-mask alignment should be made on a source-by-source basis. Furthermore, since the sources are neither parallel nor pointlike, the

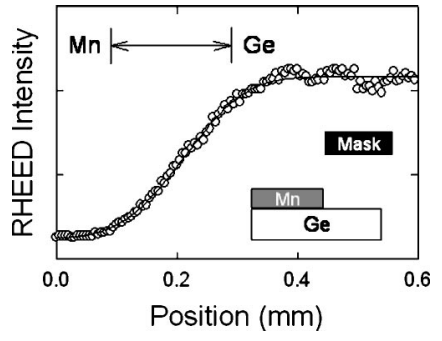

FIG. 4. Shadow profile determined by scanning RHEED, RHEED intensity versus position across an edge of Mn film deposited on Ge using a shadow mask. Inset, schematic diagram of the mask, Mn film and Ge substrate. The substrate to mask distance is $5 \mathrm{~mm}$, and the substrate to source distance is $50 \mathrm{~cm}$, and the source is about $2 \mathrm{~cm}$ in diameter. The resulting spread is $\sim 0.2 \mathrm{~mm}$. The RHEED beam is $\sim 50 \mu \mathrm{m}$ in diameter.

edge of a shadow would have a finite spread that would limit lateral dimension of the feature to be deposited. In what follows we discuss these, and in particular how to determine the actual position and profile of the shadow.

The substrate-mask alignment can be made using a video camera mounted on one of the bottom viewports of the MBE system, as shown in Fig. 1. The video system should have a sufficient optical magnification and working distance to resolve lateral dimensions on the substrate that are comparable to the spread of the shadow edge, typically better than $0.1 \mathrm{~mm}$. This dimension corresponds to a relative composition control of $\sim 1 \%$ over a typical $10 \mathrm{~mm}$ wide combinatorial sample. The actual compositional resolution can be substantially better if samples with narrower range of compositions within a phase diagram are produced and studied. The optical shadow made by a hot and bright source can be measured directly to the resolution of the video system, since the counterparts for the stepper motor controlled masks are normally much higher.

It is important to have an independent means to measure directly the edge location and profile of the feature deposited on the substrate. In-situ scanning RHEED can be used to do this, as illustrated in the case of Mn deposited on Ge shown in Fig. 4. The RHEED beam can be aligned with the edge of the mask and scanned across the edge of the deposited film with the diffraction patterns imaged and recorded by a CCD camera (see Fig. 1). The RHEED patterns can be analyzed to determine the spatial extent of the film edge, as shown in the RHEED intensity profile in Fig. 4. The position of the edge can be determined from those of the two edges of the substrate or other fiduciary features on the substrate, such as scribe marks and sample mounting clips. The size of the RHEED beam can be measured by scanning it across a sharp edge. The alignment of the beam with respect to the mask can be determined by rocking the azimuthal angle while scanning across the film edge, and minimum spread in the apparent film edge would indicate the best alignment. Since the spatial extent of the feature deposited is essentially determined by geometric factors, i.e., lateral size of the source, sample to mask and sample to source distances, by reducing the sample to mask distance, its sharpness can be enhanced accordingly. In the example shown in Fig. 4, reducing sample to mask distance from $5 \mathrm{~mm}$ to $2.5 \mathrm{~mm}$ will cause a 

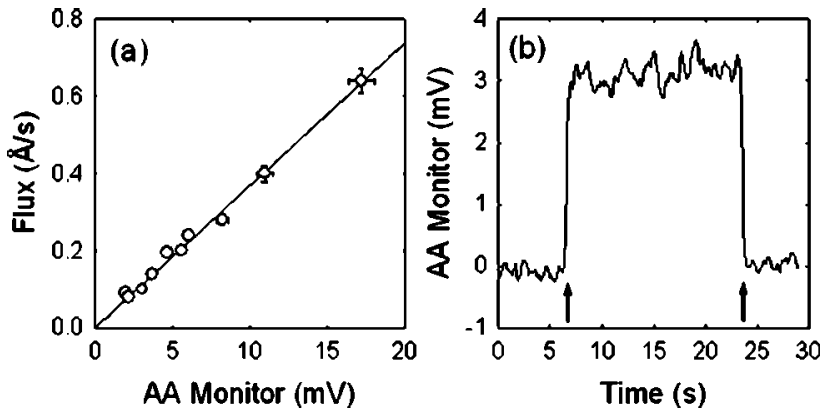

FIG. 5. Responses of a Ge AA monitor with the Ge flux from an e-beam hearth. (a) Ge flux measured by quartz crystal monitor (calibrated by RHEED specular intensity oscillations) versus absorption at a wavelength of $268 \mathrm{~nm}$. (b) Absorption versus time during deposition at a Ge flux of $0.11 \AA / \mathrm{s}$. The conversion factor for absorption is $100 \mathrm{mV}$ to $1 \%$. The arrows indicate opening and closing of the Ge shutter, and the rise/fall time corresponds to the time constant of the AA monitor.

corresponding change in the observed spread from $0.2 \mathrm{~mm}$ to $0.1 \mathrm{~mm}$.

\section{Real-time flux monitor and control}

Combinatorial deposition control requires an advanced computerized feedback system based on an array of real-time flux monitors. Conventional MBE systems are equipped with quartz crystal monitors and ion gauges that are not element sensitive and exhibit large thermally or ion induced transients caused by opening/closing of source shutters. Element sensitive flux detectors include residual gas analyzers (RGA) and AA monitors. The former are very sensitive to extremely low flux, but are prone to significant drift in the measured flux caused by direct deposition on the ionizer assembly leading to change of the work function and even electrical shorts. The latter have all the necessary attributes to perform sensitive real-time flux measurements used for real-time feedback control of the deposition process involving multiple sources. It also does not require any hardware to be placed in

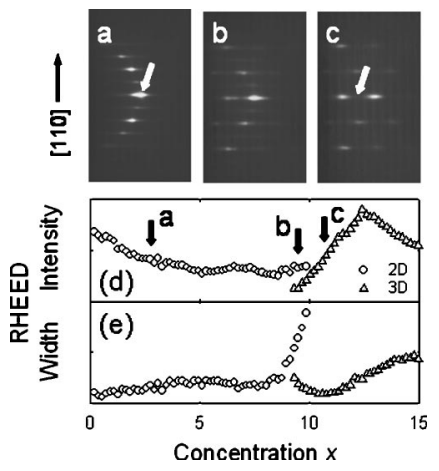

FIG. 6. Distinct RHEED patterns of $\mathrm{Co}_{0.7 x} \mathrm{Mn}_{0.3 x} \mathrm{Ge}_{1-x}$ epitaxial films as a function of doping concentration $x$ across the Ge substrate indicating the presence of a $2 \mathrm{D}$ to $3 \mathrm{D}$ roughening transition, (a) a 2D surface, (b) a mixed 2D and 3D surface, and (c) a 3D surface. Specular reflection, as indicated by the arrow in (a), was positioned between two Bragg reflections, the "antiBragg" position, as indicated by the arrow in (c). Integrated RHEED intensity and width versus $x$ are shown in (d) and (e), respectively. Circles are for the specular intensity and width of a 2D surface, and triangles are the corresponding zeroth order feature of a 3D surface. The arrows in (d) indicate the respective compositions where the RHEED patterns were taken.
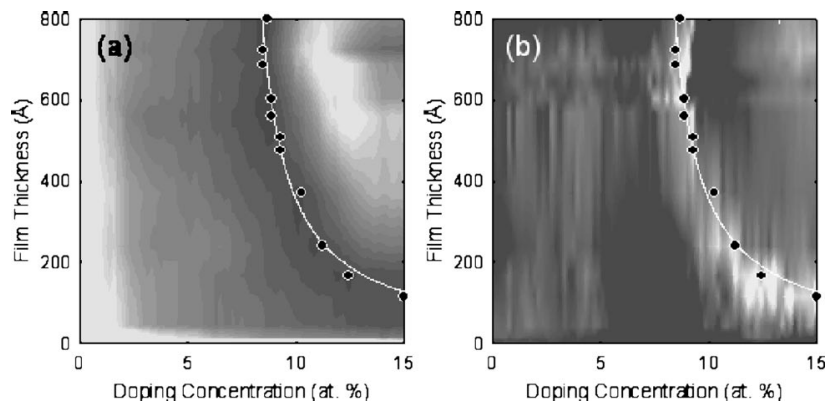

FIG. 7. Scanning RHEED to examine evolution of the roughening transition in $\mathrm{Co}_{0.7 x} \mathrm{Mn}_{0.3 x} \mathrm{Ge}_{1-x}$, RHEED intensity (a) and width (b) as a function of doping concentration and film thickness. The 2D and 3D intensities correspond to integrated intensities of the specular reflection at the anti-Bragg position and a nearby Bragg reflection, respectively (as shown in Fig. 6). Circles indicate the crossover points between the $2 \mathrm{D}$ and $3 \mathrm{D}$ features, and lines correspond to a fit based on effects of strain and phase separation induced roughening/disorder transition (Ref. 22).

the flux, unlike most flux monitors, including the ones mentioned above that are susceptible to unwanted shadow or build-up or clogging effects.

AA flux monitors use AA spectroscopy to detect and quantify atomic flux during evaporation by passing atomic emission in the UV through the vapor in front of the sample, as shown in Fig. 1 by the multicolored collimated beams. The technique was proposed 40 years ago, ${ }^{16}$ and it has since been fully developed for nearly all evaporation techniques including MBE. ${ }^{17-19}$ It is element specific that requires a separate emission/detection channel for each element. Each channel consists of a hollow cathode lamp (HCL) for the element and a modulated power supply to provide the specific atomic emission, a set of UV emitting and receiving optics including a pair of opposing quartz viewports, and a monochromator for selecting the specific AA line(s) for lock-in detection. It is sufficiently sensitive to detect flux $\geqslant 0.01 \AA / \mathrm{s}$ with a $100 \mathrm{~ms}$ time constant for nearly all atomic sources with low vapor pressure. The real-time sensitivity is demonstrated in Fig. 5 for $\mathrm{Ge}$, which is one of the least sensitive elements for AA, owing in part to the weak emission/absorption lines.

Multiple channels of AA monitors can be bundled together and mounted on one pair of opposing optical viewports, such that the deposition rates of several materials can be monitored simultaneously (Fig. 1). This provides an efficient and cost-effective approach to overcome the limited number of opposing viewports in most MBE systems, instead of occupying a large number of them. The AA monitors do require separate calibrations using in-situ techniques, including RHEED intensity oscillations and quartz crystal monitors, and ex-situ microscopy/spectroscopy techniques, including the commonly used profilometry, x-ray reflectivity and fluorescence spectroscopy, ${ }^{14,15,20}$ transmission electron microscopy, and Rutherford backscattering spectroscopy.

\section{Real-time characterization and scanning RHEED}

One of the critical characteristics of MBE is its in-situ capabilities for characterizing the properties in real time. This must be preserved and enhanced in combinatorial MBE 
in order to provide critical real-time feedback, such as what grows and under what conditions. Otherwise the synthesis process would be "blind," such that the unknown part of the parameter space would be unnecessarily large, particularly during synthesis. In general imaging and scanning techniques are compatible with combinatorial approach. The basic requirements are long working distance in order to avoid obstructing the growth process, and high spatial resolution that is compatible with the intended resolution for the variables, typically better than $0.1 \mathrm{~mm}$ on the substrate. Realtime scanning RHEED diffraction and spectroscopy satisfy these requirements, as discussed below.

Typical RHEED beams can be focused to tens of $\mu \mathrm{m}$ (higher quality ones can potentially be focused down to sub $\mu \mathrm{m}$ ) with working distance of $>$ tens of $\mathrm{cm}$, and they can be deflected and pulsed using a set of deflection coils in order to establish proper diffraction conditions. The RHEED patterns can be displayed on a phosphors screen and imaged using a CCD camera (Fig. 1). An additional set of coils can be used to work with the first one to enable parallel scanning of the beam across the sample along a fixed crystallographic azimuth. ${ }^{6,7,21}$ Structural evolution can be studied systematically in real time, as illustrated in Figs. 6 and 7 for the growth of a binary sample containing Co and Mn doped Ge (100). ${ }^{8,22}$ The RHEED patterns across a binary sample (as shown in Fig. 6) and their dependences on thickness (as shown in Fig. 7), temperature, and time can be imaged and recorded using a CCD system and analyzed. Structural and morphological transitions can be identified and examined systematically. These capabilities, not available to conventional MBE, significantly enhance the ability to probe phase transitions and phase boundaries. The imaging and scanning approaches can be adapted to a variety of techniques, particularly optical ones, including spectroscopic ellipsometry and magnetooptic Kerr effect measurements.

\section{ACKNOWLEDGMENT}

The work was supported in part by NSF Grant No. DMR-0108605.

${ }^{1}$ Committee on Condensed-Matter and Materials Physics, National Research Council, Condensed-Matter and Materials Physics: Basic Research for Tomorrow's Technology (National Academy Press, Washington, DC, 1999).

${ }^{2}$ X. D. Xiang, X. D. Sun, G. Briceño, Y. Lou, K. A. Wang, H. Chang, W. G. Wallace-Freedman, S. W. Chen, and P. G. Schultz, Science 268, 1738 (1995).

${ }^{3}$ G. Briceno, H. Chang, X. Sun, P. G. Schultz, and X.-D. Xiang, Science 270, 273 (1995).

${ }^{4}$ X. D. Xiang, Annu. Rev. Mater. Sci. 29, 149 (1999).

${ }^{5}$ T. Fukumura, M. Ohtani, M. Kawasaki, Y. Okimoto, T. Kageyama, T. Koida, T. Hasegawa, Y. Tokura, and H. Koinuma, Appl. Phys. Lett. 77, 3426 (2000).

${ }^{6}$ I. Takeuchi, R. B. van Dover, and H. Koinuma, MRS Bull. 27, 301 (2002).

${ }^{7}$ Y. K. Yoo and F. Tsui, MRS Bull. 27, 316 (2002).

${ }^{8}$ F. Tsui, L. He, L. Ma, A. Tkachuk, Y. S. Chu, K. Nakajima, and T. Chikyow, Phys. Rev. Lett. 91, 177203 (2003).

${ }^{9}$ F. Tsui, L. Ma, and L. He, Appl. Phys. Lett. 83, 954 (2003).

${ }^{10}$ Y. Matsumoto, H. Koinuma, T. Hasegawa, I. Takeuchi, F. Tsui, and Y. K. Yoo, MRS Bull. 28, 734 (2003).

${ }^{11}$ J. C. Bean, L. C. Feldman, A. T. Fiory, S. Nakahara, and I. K. Robinson, J. Vac. Sci. Technol. A 2, 436 (1984).

${ }^{12}$ F. Tsui and C. P. Flynn, Phys. Rev. Lett. 71, 1462 (1993).

${ }^{13}$ I. Bozovic, IEEE Trans. Appl. Supercond. 11, 2686 (2001).

${ }^{14}$ Y. Chu, A. Tkachuk, S. Vogt, P. Ilinski, D. Walko, D. Mancini, E. Dufresne, L. He, and F. Tsui, Appl. Surf. Sci. 223, 175 (2004).

${ }^{15}$ Y. Yamamoto, R. Takahashi, Y. Matsumoto, T. Chikyow, and H. Koinuma, Appl. Surf. Sci. 223, 9 (2004).

${ }^{16}$ W. C. Kreye, J. Appl. Phys. 35, 3575 (1964).

${ }^{17}$ T. Y. Kometani and W. Wiegann, J. Vac. Sci. Technol. 12, 933 (1975).

${ }^{18}$ M. E. Klausmeier-Brown, J. N. Eckstein, I. Bozovic, and G. F. Virshup, Appl. Phys. Lett. 60, 657 (1992).

${ }^{19}$ C. Lu and Y. Guan, J. Vac. Sci. Technol. A 13, 1797 (1997).

${ }^{20}$ S. Vogt, Y. Chu, A. Tkachuk, P. Ilinski, D. Walko, and F. Tsui, Appl. Surf. Sci. 223, 214 (2004).

${ }^{21}$ H. Koinuma, T. Koida, T. Ohnishi, D. Komiyama, M. Lippmaa, and M. Kawasaki, Appl. Phys. A: Mater. Sci. Process. 69, S29 (1999).

${ }^{22}$ F. Tsui, L. He, A. Tkachuk, S. Vogt, and Y. S. Chu, Phys. Rev. B 69, 081304R (2004) 